\title{
Image analysis of the axonal ingrowth into poly(d,l-lactide) porous scaffolds in relation to the 3-D porous structure
}

\author{
S. Blacher ${ }^{(1),(4)}$, V. Maquet ${ }^{(2),(4)}$, F. Schils ${ }^{(3),(4)}$, D. Martin ${ }^{(3),(4)}$, J. Schoenen ${ }^{(4),(5)}$, G. Moonen ${ }^{(6)}$, \\ R. Jérôme ${ }^{(2),(4)}$, J.-P. Pirard ${ }^{(1)}$ \\ (1) Department of Chemical Engineering, University of Liège, Sart-Tilman B6, 4000 Liège, \\ Belgium \\ (2) Center for Education and Research on Macromolecules, University of Liège, Sart-Tilman \\ B6, 4000 Liège, Belgium \\ (3) Department of Neurosurgery, University Hospital, CHU, B35, 4000 Liège, Belgium \\ (4) Interfacultary Center for Biomaterials, University of Liège, Sart-Tilman B6, 4000 Liège, \\ Belgium \\ ${ }^{(5)}$ Center for Cellular and Molecular Neurobiology, University of Liège, rue de Pitteurs, 20, \\ 4020 Liège, Belgium \\ ${ }^{(6)}$ Department of Neurology, University Hospital, CHU, B35, 4000 Liège, Belgium
}

\begin{abstract}
Porous polymer scaffolds are promising materials for neural tissue engineering because they offer valuable three-dimensional (3-D) supports for the in vitro and in vivo axonal growth and tissue expansion. At the time being, how the in vivo neuronal cell development depends on the scaffold 3-D architecture is unknown. Therefore, scanning electron micrographs of longitudinal sections of porous polylactide scaffolds and immunohistological sections of these scaffolds after implantation and neurofilament staining have been studied by image analysis. Pore orientation and axonal ingrowth have been investigated by spectral analysis on gray level SEM images. Binary image processing has been carried out and the binary images have been studied by spectral analysis in order to estimate the possible effect of the image noise on the real pattern. In addition to axonal orientation, density and length distribution of the regenerated axons into the polymer scaffold have been measured. Dependence of the axonal ingrowth on the 3D-polymer scaffold has been discussed on the basis of the collected data.
\end{abstract}

Keywords: Porous polymer scaffolds; Axonal growth; Image analysis; Anisotropy

\section{Introduction}

Tissue engineering is an emerging interdisciplinary research field that aims at developing new therapeutic strategies for the repair or reconstruction of damaged or lost tissues and organs $[1,2]$. Porous polymer scaffolds are promising materials for tissue engineering because they offer a physical, three-dimensional (3-D) support, as an analog of the extracellular matrix (ECM), for in vitro and in vivo cell growth and tissue expansion [3]. Design of this ECM substitute is critical for the success of tissue regeneration. During the last few years, some of us have focussed on the design and preparation of macroporous, biodegradable polymer scaffolds suited to neural tissue engineering [4-6]. Very recently, highly oriented macroporous polylactide scaffolds prepared by freeze-drying, have proved efficiency in axonal regeneration both in the peripheral and central nervous systems [7,8]. A polymer construct of ca. 20 PLA rods containing a-FGF and assembled with fibrin glue, was used to bridge the extremities of a transected spinal cord. Cell migration and angiogenesis were observed and the expected 
orientation of axonal growth, as well. The axons were indeed perfectly aligned along the pore direction, which confirmed the crucial role of the 3-D polymer structure [8]. Although the role of the ECM in the spatial architecture, differenciation and cytoskeletal organization of neural cells is known to be vital during fetal development and after nerve injury [9], the influence of the macro- and microstructure of synthetic scaffolds is on the neural cell function not currently understood. One major reason is the poor knowledge of the 3D-scaffold architecture. In this respect, some of us have reported recenly that image analysis of SEM micrographs and impedance spectroscopy are two well-suited non-destructive methods for the characterization of the texture of macroporous polylactide foams prepared by freeze-drying $[9,10]$.

The purpose of this study is to explore whether valuable information about the axonal ingrowth and orientation into polymer scaffolds can be collected by image analysis methods. So, spectral analysis has been used to characterize pore orientation on gray level images. Because these images show a complex structure, whose real pattern can be blurred by unavoidable superposed noise, binary image processing has been carried out, and the binarized images have been subjected to spectral analysis. The same methodology has been used to establish relationship between the 3D structure of the polymer scaffold and the neural cell extension and axonal orientation after immunostaining of neurofilaments (NF). Finally, relevant cellular parameters, such as axonal density and length distribution of the regenerated axons have been extracted from the binarized images of NF.

\section{Materials and methods}

\subsection{Preparation and implantation of polymer scaffolds}

Porous polymer scaffolds containing a-FGF were prepared by freeze-drying a solution of a 90:10 w:w mixture of poly(d,1-lactide) PLA (Resomer ${ }^{\circledR}$ R 206, Mn: 50,000, BoehringerIngelheim, Germany) and poly(D,L-lactide- $b$-ethylene oxide) copolymer (Mn: 10,000) in dioxane. The copolymer was synthesized as reported elsewhere [8]. The in vivo implantation of the polymer scaffold in the rat spinal cord was detailed elsewhere [8]. Briefly, a $3 \mathrm{~mm}$ length tissue gap was filled with polymer rods $(n=14-20)$, that were assembled by a-FGFcontaining fibrin glue. Rats were allowed to survive from 7 days to 12 weeks before being perfused. After removal and fixation of the spinal cord, $40 \mathrm{~mm}$ thick sections of the transplanted area were prepared and stained with different antibodies for immunohistochemical observations [8]. Immunostaining with the neurofilament antibody protein (NF)$200 \mathrm{kDa}$ (Boeh-ringer Mannheim, 1/1000) was used to study the scaffold colonization by regenerated axons.

\subsection{Microscopy}

Images were acquired from SEM micrographs of the polymer scaffolds and from optical micrographs of immunohistochemical sections in the implantation area.

Longitudinal sections of the scaffolds were prepared with a razor blade, mounted on an aluminum stub with carbon adhesive and then coated with platinum (120 s, Argon atmosphere) before observation with a Jeol JSM-840A SEM operating at an accelerating voltage of $20 \mathrm{kV}$. Five images of several areas of the same polymer scaffold were analyzed. SEM images were digitized on a matrix of 1024 x 1024 pixels with 256 gray levels. The immunostaining of NF was observed with a Zeiss (Axioplan) optical microscope at a $5 \mathrm{x}$ magnification. Optical images were digitized on a matrix of $760 \times 570$ pixels with 256 gray levels. For the sake of comparison, the images were collected with a video camera in such a 
way that the main orientation of the scaffold edges and that one of the axons were aligned in the vertical direction.

\subsection{Image analysis}

Image treatment and statistic analysis were performed with a WorkStation Sun SPARC30, and the software 'Visilog 5.0' from Noesis.

\subsubsection{Image processing}

Preliminary gray level image transformations and binary image processing were performed for both the SEM micrographs and the optical images as follows:

SEM micrographs: (a) enhancement of local pore edges by the gradient technique, (b) threshold transformation in order to binarize images, (c) opening transformation with segments oriented in the main direction of the pore edges in order to eliminate artifacts, (d) image reconstruction from open image markers.

Optical images: (a) application of a median filter to smooth background, (b) threshold transformation in order to binarize images, (c) opening transformation with a disk in order to eliminate artifacts, (d) image reconstruction from open image makers.

Total axon length per unit area $\left(\lambda\right.$ in $\left.\mathrm{mm}^{-1}\right)$ and distribution of axon length, defined as the axon length between two successive axonal crossings were estimated after transformation of the binarized images of axons with the algorithm proposed by Soille [15] which allows to separate the elongated objects that intersect one each other. Briefly (a) axons were reduced to their midline by skeletonization (b) crossing points were then dilated and suppressed from the skeleton (c) the skeleton by influence zones was calculated, (d) crossing axons were separated by the skeletal lines remaining within the original axons, (e) each segment of axon was coded with a different color and its length was automatically measured.

\subsubsection{Spectral analysis of gray level and binary images}

Pore orientation and axonal orientation in polymer scaffolds were characterized by spectral analysis of gray level and binary images. Let us briefly recall the fundamentals of this technique.

A physical 2D image is expressed as a function $f(x, y)$, such that gray levels are fluctuations of $f(x, y)$. A standard technique to identify and to quantify orientation and/or periodicity of $f(x, y)$ is to determine the Fourier power spectrum $S(\bar{k})$, which is defined in the frequency or $\bar{k}$ space as $S(\bar{k})=|F(\bar{k})|^{2} /|\bar{k}|$

$F(\bar{k}) \propto \int f(\bar{r}) \exp (-2 \pi \bar{k} \cdot \bar{r}) \mathrm{d} \bar{r}$ with $\bar{r}=(x, y)$, are the Fourier coefficients of $f(x, y)$ [11]. The frequency space representation of a pure sinusoidal structure with an orientation $\theta$, consists of two points, located on each side of the $2 \mathrm{D}$ power spectrum center, at a distance which corresponds to the wavelength of the original image with the orientation $\theta+\pi / 2$.

Superimposition of several periodic structures in the spatial domain results in the addition of the constitutive components in the frequency domain. A plot of the normalized power spectra averaged over all directions $0<\theta<2 \pi,\langle S(\bar{k})\rangle_{\theta}$ as a function of frequency gives a profile with various peaks at the characteristic frequency of each superimposed sinusoid. If the pattern is not periodic or noisy, the main frequencies are hidden by a halo which is as dense as the structure is complex. In this case, $S(\bar{k})$ should indicate the general orientation of the pattern, although in most cases the interpretation of the 2-D power spectrum is a problem. Usually, $\langle S(\bar{k})\rangle_{\theta} \propto 1 /|\bar{k}|^{\beta} . \beta=0$ means that the equal power is the same at all frequencies (white noise). $\beta=1$ is characteristic of a random structure in which the altitude variations 
$\Delta f(x, y)$ are completely uncorrelated (pink noise). $\beta=2$ is typical of a smooth surface. The fractal dimension, $D$; is related to $\beta(D=(6-\beta) / 2)$.

For anisotropic surface images, the slope of $\log S(\bar{k} x)$ vs $\log$ frequency as a function of the direction, i.e., a rose plot, allows degree and direction of anisotropy and surface fractal dimension to be evaluated $[12,13]$. Surface structure observed by scanning tunneling microscopy (STM) and atomic force microscopy (AFM) have been characterized by this technique $[12,13]$.

\section{Results}

Two populations of pores are observed in the porous scaffolds prepared by freeze-drying for in vivo implantation, i.e. macropores of ca. $100 \mathrm{~mm}$ that coexist with ca. 10 times smaller pores. From transverse SEM micrographs (not shown here), the characteristic pore sizes were measured by image analysis using the methodology reported elsewhere $[9,10]$. Density and diameter of the macropores were 0.176 and $0.09170 .027 \mathrm{~mm}$, respectively. Density and average area of the micropores 0.60 and $1.58270 .206 \mathrm{~mm}^{2}$, respectively.

In this paper, image analysis was used to characterize the orientation of the pore edges and the direction of the axons that grew in these porous scaffolds 1 month after implantation.

\subsection{Polymer scaffolds}

Figs. 1a and b show two representative SEM micrographs of highly oriented PLA scaffolds intended for spinal cord regeneration. For the sake of comparison, SEM micrograph of a porous PLA sample with no well-defined porous structure is reported in Fig. 1c. Figs. 1d-f are the 2D power spectra of the SEM micrographs shown in Figs. 1a-c, respectively, and drawn in a perspective view, with the logarithmic values of the power intensities. The cross pattern of horizontal and vertical lines, which appear in all cases, is an artifact due to the assumption that the processed 2D signal is periodic. This feature has been ignored in the analysis and further calculations.

The power spectra shown in Figs. 1d and e are patterns oriented along the line $\theta=0$, which corresponds to SEM images in which most pore edges are nearly vertically oriented. The flat structures are surrounded by a halo that hides the frequencies related to the characteristic lengths of the original SEM images. In contrast, the power spectrum for the SEM morphology reported in Fig. 1c is rather isotropic, with all the excited frequencies distributed in a central circle and surrounded by a halo of a decreasing density (Fig. 1f).

The rose plots reported in Figs. 1g-i give information on the degree of anisotropy of the pore structures shown in Fig. 1a-c, depending on the direction. For being comparable, all the graphs have been normalized. The rose plot is quasi circular in case of the isotropic morphology shown in Fig. 1c. For the anisotropic structures (Figs. 1a and b), the slope gradually changes with the direction and results in a non-circular pattern (Figs. 1g and $\mathrm{h}$, respectively). The rose plot for the porous structure shown in Fig. 1a, indicates that the majority of edges are oriented in the direction $\theta=\pi / 2$; whereas for the structure shown in Fig. $1 \mathrm{~b}$, the rose pattern also shows a main orientation at $\theta=\pi / 3$. From the direct observation of Figs. 1a and $\mathrm{b}$, it appears that the orientation $\theta=\pi / 2$ accounts for most of the largest channels of the macroporous structure, whereas the other directions refer to the edges within these channels (the microporous ladder-like structure). 

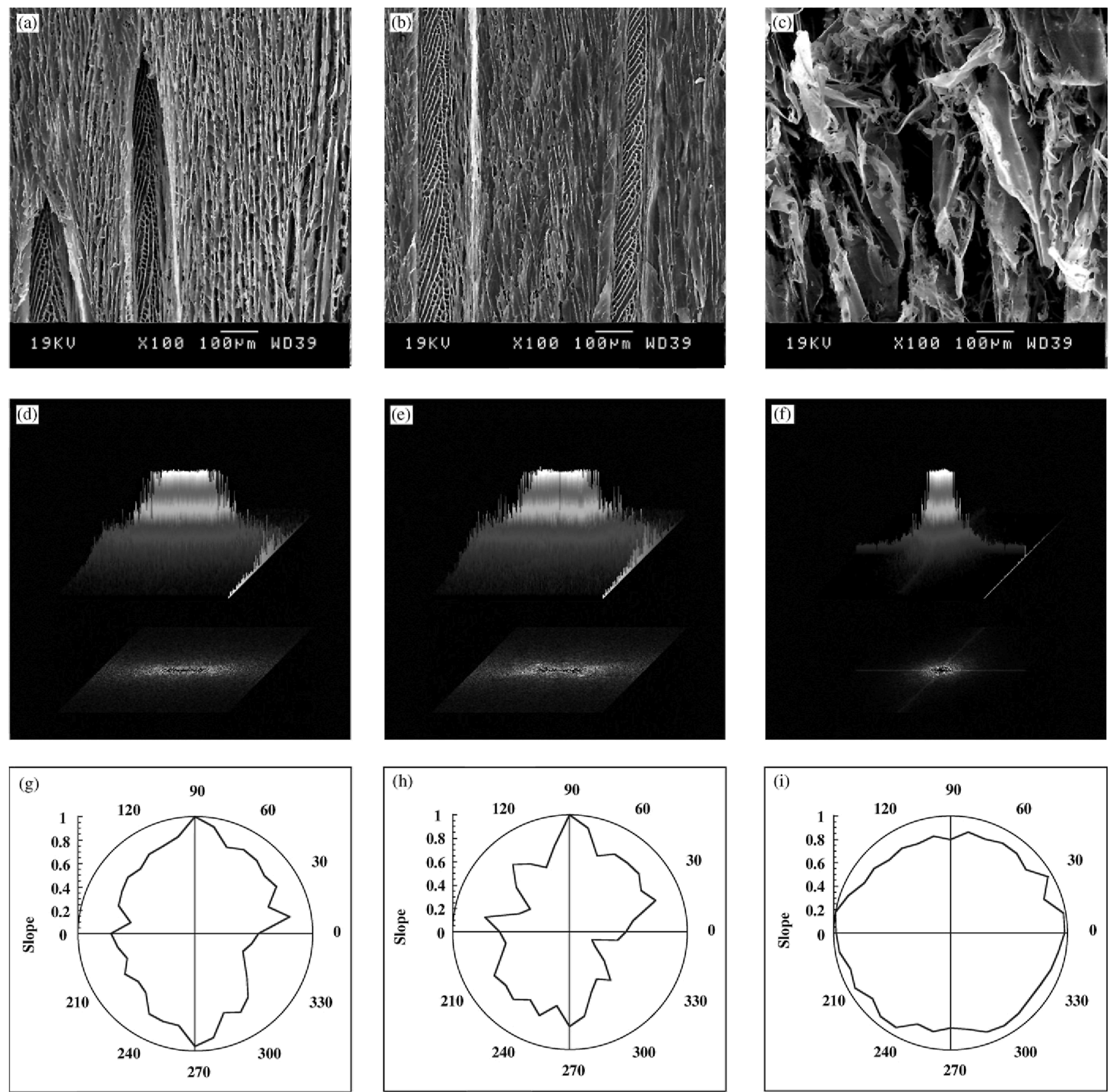

Fig. 1. SEM micrographs of longitudinal sections of PLA scaffolds with highly oriented pores

(a) and (b), and no well-defined porous structure (c) at a $\boldsymbol{x} 100$ magnification. (d)-(f) $2 D$ power spectra, drawn in a perspective view for samples a-c. (g)-(i) Normalized rose plots of the SEM micrographs shown in Fig. 1 (a)-(c).

Gray level images show a complex structure in which, either an uneven illumination, or a lack of contrast or other sources of noise, hide the real pattern. In order to estimate the influence of noise on the analysis of anisotropy, pore edges have been extracted from the gray level images and spectral analysis has been carried out for the binary images. Figs. $2 \mathrm{a}$ and $\mathrm{b}$ show the images that result from the binarization of Figs. 1a and b, respectively. It must be noted that the power spectra of these binary images (Figs. 2c and d) are flatter than those of the parent gray level images (Figs. 1d and e) because noise elimination decreases the intensity and size of the halo. Nevertheless, the halo persists because the pattern is not really periodic. Comparison between Figs. 1g,h and 2e,f indicates that the pattern of the rose plots extracted 
from the binary and gray level images is similar with, however, a less smooth contour for the rose plots associated to the binary images.

\subsection{Axonal growth}

Figs. $3 \mathrm{a}$ and $\mathrm{b}$ show two representative images of NF-immunostaining in the polymer implant, 1 month after implantation. Figs. $3 \mathrm{c}$ and $\mathrm{d}$ are the binarized images that result from the same processing image as before. The rose plots (Figs. $3 \mathrm{e}$ and $\mathrm{f}$ ) indicate that the axons have grown in different directions with, however, a preferential orientation along the main pore direction.
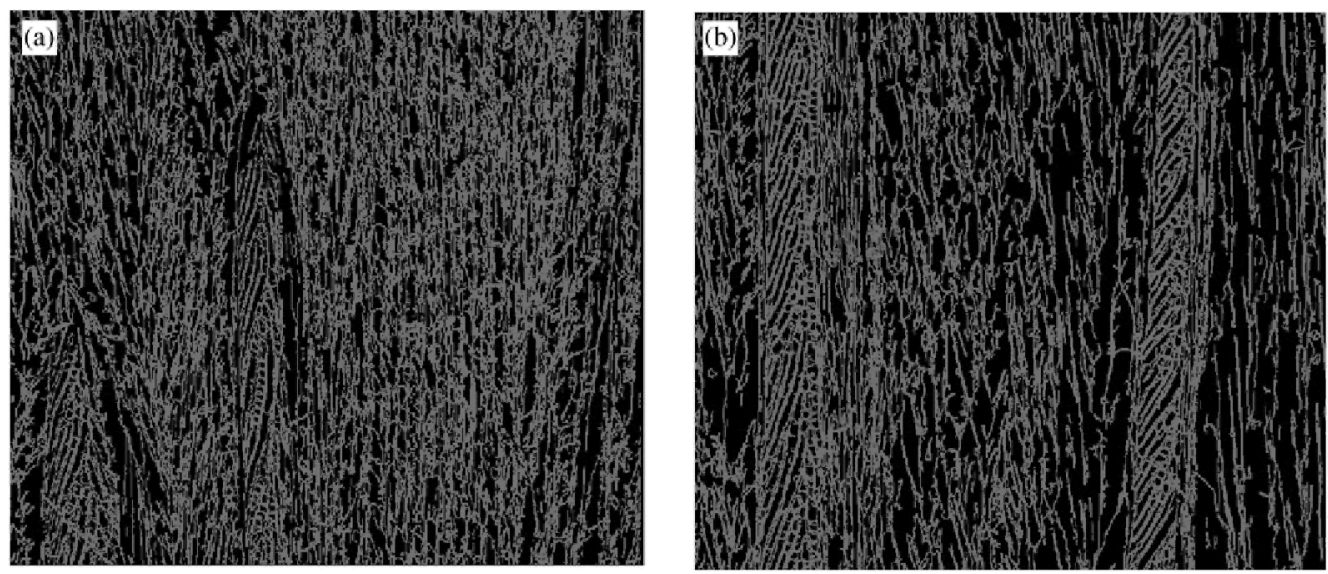

\section{(c)}
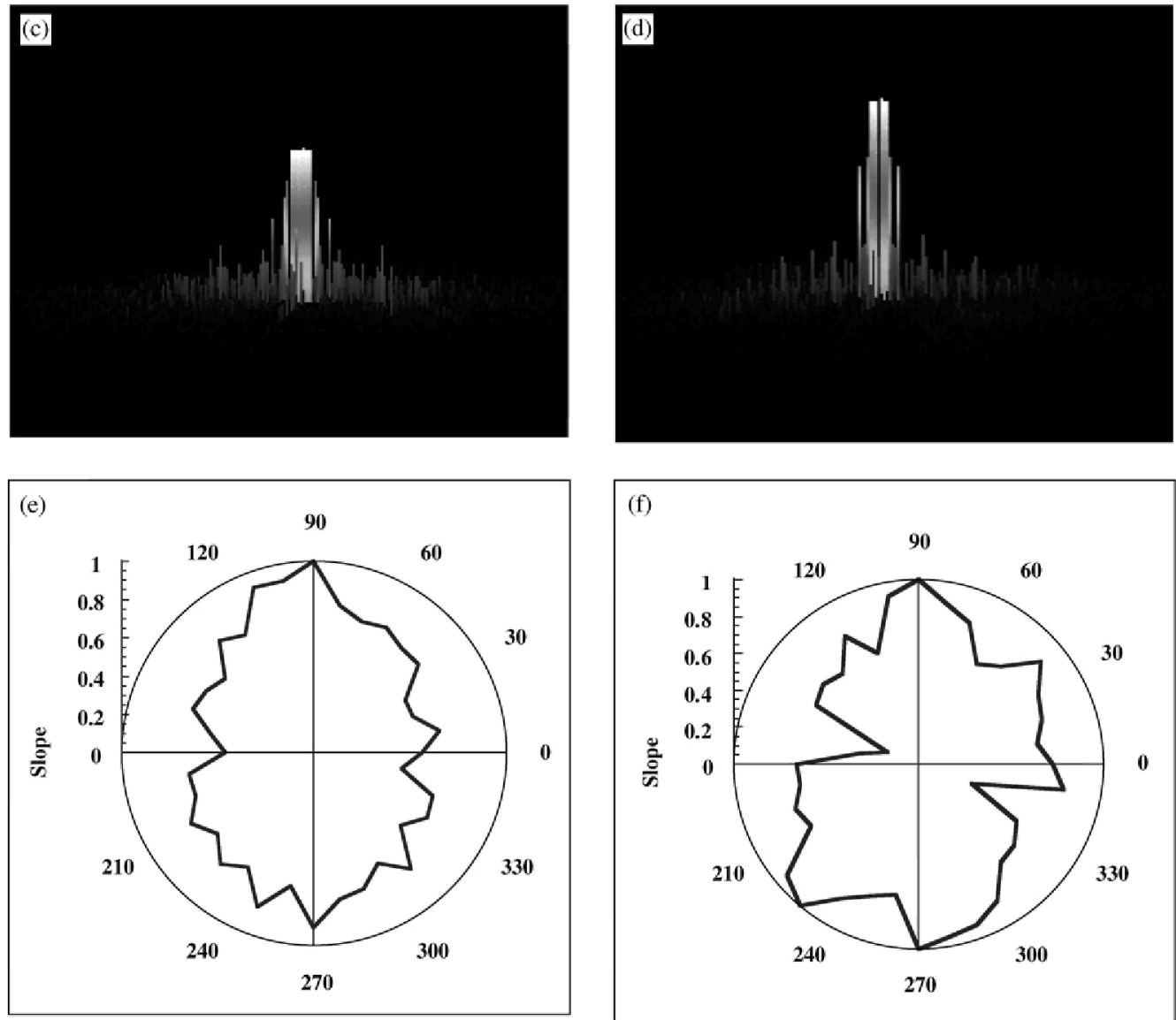

Fig. 2. (a, b) Binary images of the oriented PLA scaffold shown in Figs. 1a and b and related $2 D$ power spectra (c) and (d) and rose plots (e) and (f). 
The axonal density $\left(\lambda=2.66 \mathrm{~mm}^{-1}\right)$ has been determined from these binarized images.

Figs. $4 \mathrm{a}$ and $\mathrm{b}$ show color-coded axonal segments, i.e., the segments of axons lying between two crossings, as put forward by the procedure described in Section 2.3.1. Fig. 4c shows the histogram of the axon length and the results of the statistical analysis. It appears that: (a) the axon length is distributed over a large range from $3 \mu \mathrm{m}$ to $0.6 \mathrm{~mm}$, (b) the distribution curve fits a nearly log-normal law, with Skewness and Kurtosis values very far from 0 , typical of a distribution with a very long tail toward the highest length value. In this case, calculation of an average value would be meaningless, providing an underestimated distance between two crossing points.

\section{Discussion}

Tissue engineering in the nervous system is a major challenge because of the complex biology, chemistry and physiology of many disorders and traumatisms. Some of us started to address the challenge of spinal cord repair by using macroporous 3D-polymer implants consisting of precisely oriented PLA porous rods containing a-FGF and strongly bonded to the spinal cord by fibrin glue. As reported by Cheng et al. [14], the 3D organization of a spinal reconstruction device is vital. In order to understand better this interdependence, the scaffold porosity and anisotropy have been characterized and compared to the neurite extension. In a previous work, image analysis of SEM micrographs of transverse scaffold sections was carried out to determine the macro- and micropore characteristic sizes and shapes. 

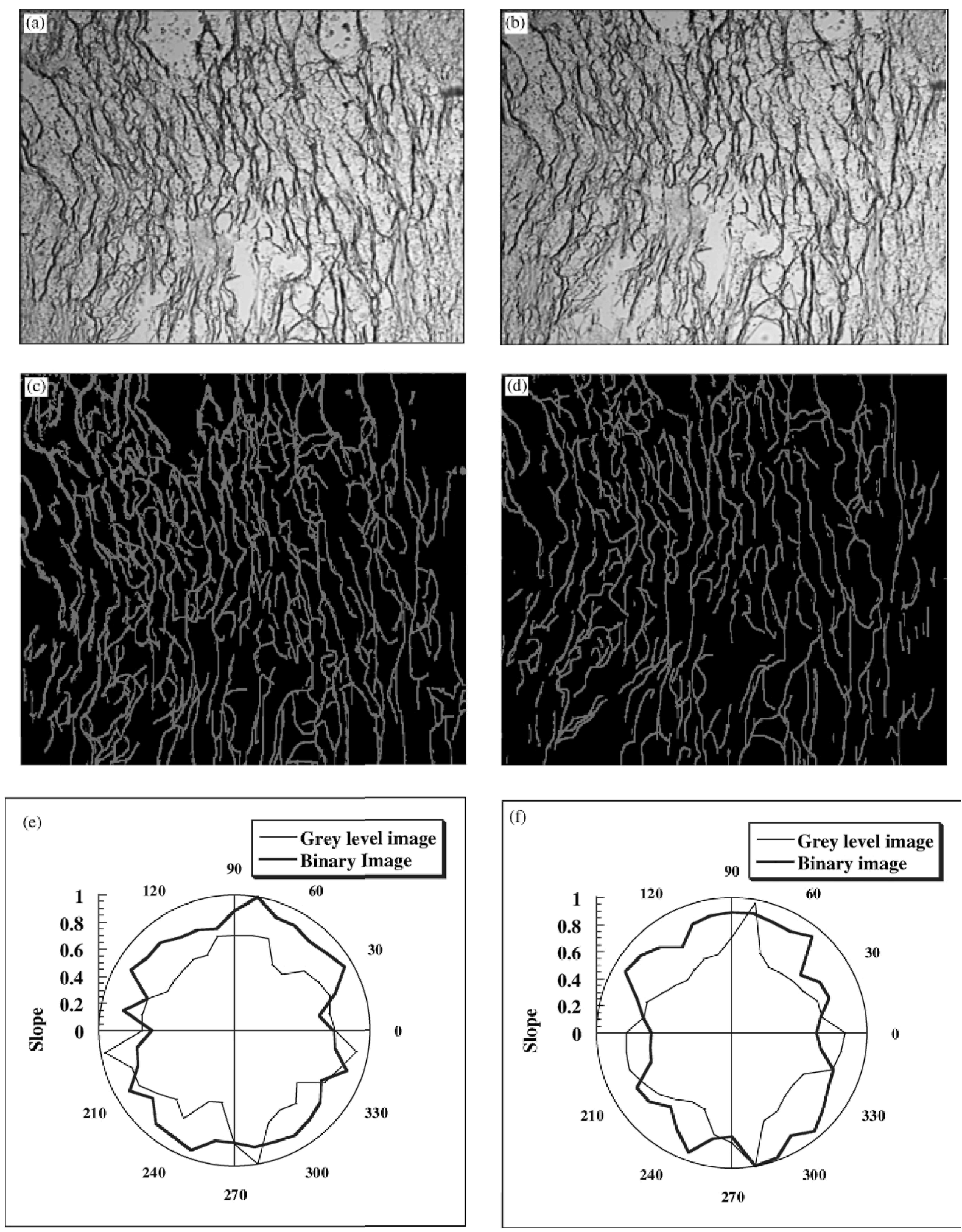

Fig. 3. (a, b) Immunohistochemical staining for neurofilaments 1 month after polymer implantation. (c) and (d) are the binarized images and (e) and (f) are the rose plots for samples $a$ and $b$. 

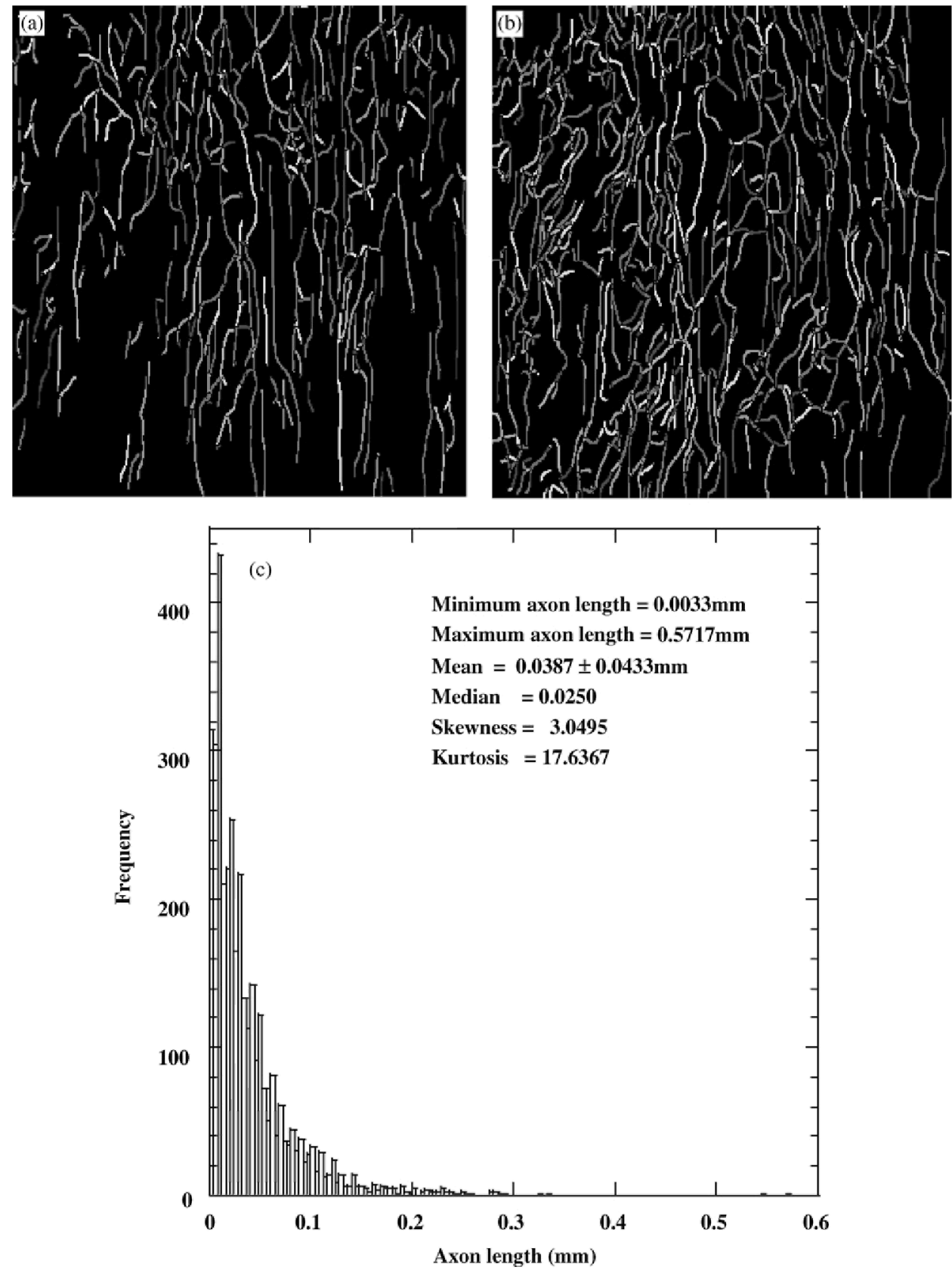

Fig. 4. (a) and (b) Axonal segmentation, (c) histogram of axon length and statistical analysis.

Moreover, longitudinal SEM sections, in which the brightness pattern depends on the porous edge orientation, were prepared to estimate the degree of anisotropy of the porous structures. Various methods are reported in the scientific literature for the analysis of this anisotropy. Mathematical morphology and spectral analysis are the most popular methods. In the first approach, a series of erosion transformations in various directions is carried out using a pair of points [15]. Then, the plot of volume versus direction of these pairs of points determines the orientation of the image structure. This technique is very sensitive to noise because all the pixel intensities are taken into account. In contrast, in the spectral analysis, the frequency noise can be ignored in the calculation of the slope of the $\log S(\bar{k})$ versus $\log \bar{k}$ plot. Anyway, even if all the frequencies are taken into account in the slope calculation, noise may only have a minor effect because of the large number of data which are plotted (512 in our application). The results reported here support that image analysis is an efficient tool to characterize the anisotropy of the porous structure observed by SEM. This method has emphasized the ability 
of the neural cells to extent their axons in a 3D porous scaffold, particularly along the main pore orientation. Comparison of the data collected from both the gray level and the binary images indicates that no information is lost when the image is binarized as proposed in this work. Moreover, binarized images have been used to quantify the axonal density, which is an indication of cell ability to invade the polymer scaffold. By using the algorithm developed by Soille (15), the overlapping axons have been separated, and the axonal length distribution has been quantified. This distribution is broad and fits a log-normal curve. The same type of distribution profile has been reported for the micropore size distribution of porous polylactide scaffolds, which emphasizes an analogy between the scaffold morphology and the cell ingrowth.

\section{Conclusions}

In this study, a methodology based on image analysis has been developed in order to analyze as quantitatively as possible the regeneration of axons into the porous structure of polylactide scaffolds. The results confirm the role of the highly oriented pore morphology in guiding axonal regeneration and validate the strategy of spinal cord repair that we previously developed. This methodology is a very promising tool to gain more insight into the relationship between the structure of the polymer scaffold and cell colonization, which is a major issue in biomaterials science and engineering.

\section{Acknowledgements}

S.B. is indebted to Action de Recherche Concertee (ARC No. 00/05-265) for financial support.

V.M. is "Post doctoral Researcher" by the "Fonds National de la Recherche Scientifique" (FNRS). CERM is indebted to the "Services Fedéraux des Affaires Scientifiques, Techniques et Culturelles" for support in the frame of the "Poles d'Attraction Interuniversitaires: PAI 4/11".

\section{References}

[1] Patrick CW, Mikos AG. In: McIntire LV, editors. Frontiers in tissue engineering. Oxford: Pergamon Press, 1998.

[2] Lanza RP, Langer R, Chick WL. Principles of tissue engineering. RG Landes and Co., Austin: Academic Press, Inc., 1997.

[3] Shoichet MS, Hubbel JA. Polymers for tissue engineering. Utrecht: VSP, 1998.

[4] Schugens C, Maquet V, Grandfils C, Jer!ome R, Teyssié P. Biodegradable and macroporous polylactide implants for cell transplantation: 1. Preparation of macroporous polylactide supports by solid-liquid phase separation. Polymer 1996;37: 1027-38.

[5] Schugens C, Maquet V, Grandfils C, Jérôme R, Teyssié P. Polylactide macroporous biodegradable implants for cell transplantation: II. Preparation of polylactide foams by liquid-liquid phase separation. J Biomed Mater Res 1996; 30:449-61.

[6] Maquet V, Jérôme R. Design of macroporous biodegradable polymer scaffold for cell transplantation. In: Liu D-M, Dixit V, editors. Porous materials for tissue engineering. Uetikon-Zuerich: Trans Tech Publications Ltd., 1997. p. 15-42.

[7] Maquet V, Martin D, Malgrange B, Franzen R, Schoenen J, Moonen G, Jérôme R. Peripheral nerve regeneration using bioresorbable macroporous polylactide scaffolds. $\mathrm{J}$ Biomed Mater Res 2000;52:639-51.

[8] Maquet V, Martin D, Scholtes F, Schoenen J, Moonen G, Jérôme R. Poly(D,L- 
lactide)foams modified by poly(ethylene oxide)-block-poly(DL-lactide) copolymers and aFGF: in vitro and in vivo evaluation for spinal cord regeneration. Biomaterials 2001; 22:1137-46.

[9] Maquet V, Blacher S, Pirard R, Pirard J-P, Jérôme R. Characterization of porous polylactide foams by image analysis and impedance spectroscopy. Langmuir 2000;16:10463-70.

[10] Blacher S, Maquet V, Pirard R, Pirard J-P, Jérôme R. Image analysis, impedance spectroscopy and mercury porosimetry characterization of freeze-drying porous materials. Colloids and Surface A 2001;187-188:375-83.

[11] Falconer K. Fractal geometry, mathematical foundations and applications. New York: Wiley, 1994.

[12] Russ JC. Surface characterization, fractal dimensions, Hurst coefficients and frequency transforms. J Comput-Assist Microsc 1990;2:161-83.

[13] Russ JC. The image processing handbook, 3rd ed.. Florida: CRC press LLC, 1999.

[14] Cheng H, Cao Y, Olson L. Spinal cord repair in adult paraplegic rats: partial restoration of hind limb function. Science 1996; 273:510-3.

[15] Soille P. Morphological image analysis. Principles and applications. Berlin, Heidelberg: Springer, 1999. 